\title{
Web DUE: Uma Técnica de Inspeção de Usabilidade de Mockups de Aplicações Web Guiada Por Zonas Próprias de Páginas Web
}

\author{
Luis Rivero e Tayana Conte
}

\author{
Programa de Pós-Graduação em Informática \\ Instituto de Computação - Universidade Federal do Amazonas (UFAM) \\ Av. Rodrigo Otávio, $\mathrm{n}^{\circ}$ 6.200. CEP: 69.077 - 000, Manaus - AM - Brazil \\ \{luisrivero, tayana\} @icomp.ufam.edu.br
}

\begin{abstract}
Usability is one of the key factors for software quality that affects the acceptance of the developed applications. However, despite the increasing number of usability inspection methods for Web applications, most of the proposed methods can only be applied in later stages of the development process, which increases the cost of the identified problems. In order to solve that gap, in this master's research we proposed the Design Usability Evaluation (DUE) technologies: (a) the Web DUE technique, which is specific for the usability inspection of low fidelity prototypes (or mockups) of Web applications; and (b) the Mockup DUE tool, which supports its inspection process. Besides showing how we developed these technologies, this paper presents their empirical evaluation, their evolution, and the obtained results and benefits from this research.
\end{abstract}

Resumo. Usabilidade é um dos atributos de qualidade de software que afetam a aceitação das aplicações desenvolvidas. No entanto, apesar do aumento do número de métodos de inspeção de usabilidade de aplicações Web, a maioria destes métodos só pode ser aplicada nas últimas etapas do processo de desenvolvimento, aumentando o custo de correção dos problemas identificados. Com o intuito de solucionar esta dificuldade, nesta pesquisa de mestrado foram propostas as tecnologias Design Usability Evaluation (DUE): (a) a técnica Web DUE, que é específica para inspeção de usabilidade de protótipos de baixa fidelidade (ou mockups) de aplicações Web; e (b) a ferramenta Mockup DUE que apoia sua aplicação. Além de apresentar como foram desenvolvidas estas tecnologias, este artigo apresenta a avaliação experimental das mesmas, sua evolução, e os resultados e beneficios decorrentes desta pesquisa.

\section{Introdução}

Usabilidade é um dos seis atributos de qualidade de software (ISO/IEC 25010, 2011). Resultados de estudos experimentais sobre a importância dos atributos de qualidade de software apontaram que a usabilidade foi considerada o atributo mais importante (de la Vara, 2011). Em aplicações $W e b$, a usabilidade tem um forte impacto na sua aceitação (Matera et al., 2006). Além disso, as dificuldades experimentadas pelos usuários com as aplicações Web determinam o seu sucesso ou fracasso (Fernandez et al., 2011). 
Para permitir a avaliação de atributos de usabilidade em aplicações de software, a indústria e a academia têm proposto métodos de avaliação divididos em testes com usuários e inspeções (Fernandez et al., 2011). No teste com usuários, os usuários finais realizam tarefas na aplicação, enquanto um observador checa se eles podem efetivamente e eficientemente usar a aplicação. Por outro lado, em uma inspeção de usabilidade, inspetores verificam atributos de usabilidade utilizando técnicas específicas de inspeção. Apesar de permitir a identificação de um número maior de problemas de usabilidade, um teste com usuários requer laboratórios com equipamentos específicos para medir a resposta do usuário, o que pode tornar sua aplicação custosa. Além disso, é necessário recrutar vários usuários com diferentes perfis, que podem não estar disponíveis no momento da avaliação. Esta pesquisa foca na avaliação da qualidade de software em termos de usabilidade através de inspeções devido às suas vantagens em relação ao teste com usuários.

Ao realizar uma extensão em cima de um mapeamento sistemático (Rivero e Conte, 2012a), foi possível identificar que é necessário que novos métodos de inspeção de usabilidade de aplicações Web: (a) apóiem a identificação de problemas de usabilidade nas primeiras etapas do processo de desenvolvimento deste tipo de aplicações; (b) permitam a identificação de problemas específicos e guiem o processo de inspeção; e (c) forneçam apoio ferramental, com o intuito de facilitar a inspeção e melhorar seus resultados. Esta pesquisa de mestrado teve por objetivo prover soluções para estes problemas em termos de inspeções de usabilidade de Aplicações Web.

Neste artigo são apresentados os principais resultados de uma dissertação de mestrado sobre métodos de inspeção de usabilidade de aplicações $W e b$, na qual foi proposto um conjunto de tecnologias denominadas Design Usability Evaluation (DUE). $\mathrm{Na}$ Seção 2 são apresentados: o referencial teórico base para esta pesquisa e os resultados da extensão de um mapeamento sistemático sobre métodos de avaliação de usabilidade. Na Seção 3 são descritas as tecnologias propostas: a técnica Web DUE e a ferramenta Mockup DUE; e seu processo de aplicação. A Seção 4 apresenta os diferentes estudos experimentais executados para avaliar o desempenho das tecnologias propostas e seus resultados. Finalmente, na Seção 5 são apresentadas as conclusões e trabalhos futuros decorrentes desta pesquisa de mestrado.

\section{Background}

\subsection{Inspeção de Usabilidade}

Métodos de Avaliação de Usabilidade (Usability Evaluation Methods - UEMs) são procedimentos compostos por um conjunto bem definido de atividades que são usadas para avaliar a usabilidade de um sistema (Matera et al., 2006). Segundo Fernandez et al. (2011), inspeções de usabilidade são um tipo de UEMs em que inspetores ou a equipe de desenvolvimento verificam a conformidade dos artefatos de software com aspectos de usabilidade. Com base na sua avaliação, os inspetores podem encontrar prováveis problemas de usabilidade. A principal vantagem dos métodos de inspeção de usabilidade é que requerem menos recursos e consequentemente, podem diminuir os custos de encontrar problemas de usabilidade (Fernandez et al., 2011). 
Segundo Matera et al. (2006), os métodos mais representativos de inspeção de usabilidade são: a Avaliação Heurística (Nielsen, 1992) e o Walkthrough Cognitivo (Polson et al., 1992). Na Avaliação Heurística, um conjunto de avaliadores examina a interface e categoriza defeitos de usabilidade baseado em uma lista de heurísticas. Estas heurísticas são um conjunto de regras ou diretrizes que visam descrever propriedades comuns de interfaces usáveis (Nielsen, 1992). O Walkthrough Cognitivo (CW), por outro lado, é um método que simula o processo de solução de problemas de um usuário; isto é, o que o usuário fará em determinadas circunstâncias de uso e por que (Polson et al., 1992). Durante o processo de inspeção, os inspetores executam uma tarefa para verificar possíveis problemas de usabilidade. A avaliação é feita passo a passo, enquanto o CW guia os avaliadores na análise das ações que os usuários irão realizar para atingir um objetivo em uma determinada tarefa.

\subsection{Estado Atual dos Métodos de Inspeção de Usabilidade de Aplicações Web}

Fernandez et al. (2011) executaram um mapeamento sistemático para identificar novos métodos de avaliação de usabilidade (UEMs) de aplicações Web. Com o intuito de identificar oportunidades de pesquisa nessa área, foi executada uma extensão deste mapeamento, focando nas propostas de métodos de inspeção. Nesta seção são apresentados os resultados da extensão do mapeamento sistemático cujos resultados foram publicados em trabalhos anteriores (Rivero e Conte, 2012a e Rivero et al., 2013a). Esses artigos podem ser consultados para maiores informações sobre os critérios de seleção, inclusão e exclusão, assim como a análise dos 26 artigos selecionados.

Com relação à base teórica dos métodos de inspeção para aplicações $W e b$, foi identificado que aproximadamente $46 \%$ dos artigos selecionados descreviam novos métodos de inspeção que se baseiam em métodos genéricos como a Avaliação Heurística e o Walkthrough Cognitivo. Além disso, 15\% dos artigos selecionados relatavam métodos que utilizam perspectivas próprias da $\mathrm{Web}$ para ajudar os inspetores a concentrar a avaliação em diferentes atributos de usabilidade, e assim melhorar seus resultados. Finalmente, outros artigos (58\%) relatavam técnicas que utilizam diretrizes novas e específicas para o domínio Web. O percentual de artigos pode somar mais que $100 \%$ devido a que uma técnica pode possuir mais de uma base teórica.

Os resultados desta revisão também permitiram identificar que tipos de artefatos estão sendo inspecionados. Na maioria dos artigos (77\%), os métodos de inspeção de usabilidade focam na avaliação de aplicações Web prontas ou parte funcional delas. Além disso, $15 \%$ dos artigos selecionados descrevem verificadores de código HTML que permitem executar uma inspeção de usabilidade de forma automatizada. Finalmente, $15 \%$ dos artigos selecionados relatam métodos de inspeção de usabilidade que permitem avaliar se os modelos de software seguem regras de interação no contexto $W e b$. Visto que algumas técnicas podem ser utilizadas para avaliar diferentes artefatos, a soma dos percentuais de artigos também pode somar mais que $100 \%$.

Com relação ao tipo de aplicação $W e b$ avaliado, os resultados da extensão do mapeamento sistemático permitiram identificar que alguns dos artigos selecionados (12\%) descrevem técnicas que focam na avaliação de um tipo específico de aplicação Web como: (a) médica, (b) e-commerce, e (c) Web 2.0. Nesse sentido, métodos para um tipo específico de aplicação $W e b$ permitem identificar mais problemas, porém não 
podem ser aplicados a todas as aplicações $W e b$. Além disso, dos métodos genéricos existentes, poucos permitem guiar os inspetores pelo processo de inspeção e/ou fornecer exemplos para a identificação de problemas de usabilidade.

Finalmente, o grau de apoio ferramental e automação dos métodos de inspeção desenvolvidos depende do artefato sendo avaliado. Por exemplo, a análise de código HTML está sendo totalmente automatizada, onde não é necessária a participação de um inspetor, pois as regras de usabilidade estão embutidas no analisador de código. No entanto, os problemas identificados por um analisador de código estão limitados a problemas relacionados com cores e padrões. Além disso, poucos dos métodos de inspeção possuem apoio ferramental para melhorar o desempenho dos inspetores.

\section{As Tecnologias DUE}

A extensão do mapeamento sistemático sobre métodos de inspeção de usabilidade de aplicações Web permitiu identificar oportunidades de melhoria nas atuais propostas. Com relação aos artefatos avaliados, observou-se a necessidade de permitir a inspeção de artefatos de software produzidos antes da escrita do código fonte da aplicação $W e b$; visto que o custo de correção de problemas aumenta nas ultimas etapas do processo de desenvolvimento (Travassos et al., 1999). Além disso, identificou-se a necessidade de permitir a avaliação de aplicações $W e b$ de forma genérica, porém guiando os inspetores para aumentar o desempenho da inspeção. Finalmente, com relação ao grau de apoio ferramental, percebeu-se a necessidade de desenvolver ferramentas que automatizassem parte do processo de inspeção das técnicas propostas, para facilitar o uso das mesmas.

As necessidades citadas acima foram a motivação para a criação das tecnologias Design Usability Evaluation (DUE). Nas próximas subseções são descritas cada uma das tecnologias e seu processo de aplicação para identificar problemas de usabilidade.

\subsection{A Técnica Web DUE}

A Web Design Usability Evaluation (Web DUE), é uma técnica de inspeção de usabilidade genérica de aplicações Web que tem por objetivo: (a) diminuir os custos de correção dos problemas de usabilidade identificados; e (b) orientar os inspetores no processo de avaliação. Para atingir estes objetivos, a técnica Web DUE sugere a inspeção de usabilidade de protótipos de baixa fidelidade (ou mockups) de aplicações Web. Um mockup é um modelo de software que mostra, através de imagens, como seria a aparência da aplicação Web quando o seu desenvolvimento fosse concluído. Visto que mockups podem ser desenvolvidos no início do processo de desenvolvimento, ao permitir a sua avaliação, a técnica Web DUE visa identificar problemas de usabilidade antes da escrita do código fonte da aplicação, diminuindo os custos de correção dos mesmos. Além disso, para guiar os inspetores durante a identificação de problemas de usabilidade, a técnica Web DUE sugere a divisão dos mockups em zonas de páginas Web que, segundo Fons et al. (2008), referem-se a uma parte da página Web que tem um tipo específico de conteúdo. Ao utilizar as zonas, os inspetores só deverão avaliar os atributos de usabilidade para as zonas que compõem o mockup avaliado.

A técnica Web DUE disponibiliza um conjunto de itens de verificação para cada zona de página $W e b$ presente no mockup. Estes itens descrevem propriedades específicas de usabilidade que visam apoiar os inspetores no processo de identificação de 
problemas. Os itens de verificação da técnica Web DUE foram desenvolvidos evoluindo a técnica Web Design Perspectives - WDP (Conte et al., 2009) que é uma técnica de inspeção que adapta a técnica Avaliação Heurística no contexto Web através do uso das perspectivas: apresentação, conceituação e navegação. Para facilitar o entendimento dos itens de verificação e/ou como atingir a propriedade de usabilidade, a técnica Web DUE fornece exemplos / esclarecimentos pareados com cada um dos seus itens de verificação. A Tabela 1 apresenta a descrição de uma das zonas de páginas Web da técnica Web DUE e parte dos seus itens de verificação e exemplos / esclarecimentos. A listagem completa das zonas e seus respectivos itens podem ser encontrados em um trabalho anterior (Rivero et al., 2013a).

Tabela 1. Extrato da técnica Web DUE para a zona de entrada de dados.

\begin{tabular}{|l|l|l|}
\hline \multicolumn{3}{|l|}{$\begin{array}{l}\text { Zona de Entrada de Dados: Esta zona é responsável por providenciar ao usuário um meio de entrada de dados para executar } \\
\text { determinadas operações. Posteriormente o usuário clicará em um botão que ativará uma função com base nos dados inseridos. }\end{array}$} \\
\hline ID & Item de Verificação & Exemplo / Esclarecimento \\
\hline I01 & Os campos a serem informados são legíveis. & $\begin{array}{l}\text { O nome dos campos está visível e a letra usada é fácil de ler com letras } \\
\text { suficientemente grandes e bom contraste. }\end{array}$ \\
\hline I02 & $\begin{array}{l}\text { Os dados obrigatórios na entrada de dados } \\
\text { estão claramente definidos. }\end{array}$ & $\begin{array}{l}\text { O usuário deve saber quais dados são indispensáveis para usar o sistema. } \\
\text { Por exemplo, o sistema usa "*" ou "(obrigatório)" ao lado dos campos } \\
\text { obrigatórios. }\end{array}$ \\
\hline I03 & $\begin{array}{l}\text { É possível reconhecer o significado dos } \\
\text { termos (palavras ou símbolos) utilizados. }\end{array}$ & $\begin{array}{l}\text { O usuário deve conseguir identificar que dados estão sendo solicitados } \\
\text { sem dificuldade. Por exemplo, um campo data, não devia possuir nomes } \\
\text { ambíguos como "época", "tempo", etc. }\end{array}$ \\
\hline I04 & $\begin{array}{l}\text { A interface permite a utilização de de } \\
\text { mecanismos de busca de informação que } \\
\text { auxiliem a entrada de dados obrigatória. }\end{array}$ & $\begin{array}{l}\text { Caso o usuário não tenha acesso a um dado obrigatório, o sistema deve } \\
\text { facilitar este acesso de alguma forma. Por exemplo, na hora de inserir um } \\
\text { endereço, o sistema pode permitir procurar endereços no google maps. }\end{array}$ \\
\hline
\end{tabular}

\subsection{A Ferramenta de Apoio Mockup DUE}

A ferramenta Mockup Design Usability Evaluation (Mockup DUE) foi proposta para apoiar a utilização da técnica Web DUE, reduzindo o esforço cognitivo em cima dos inspetores ao executarem uma inspeção de usabilidade (Rivero e Conte, 2013b). Para diminuir a dificuldade do inspetor de ter de simular a interação com o sistema enquanto realiza a inspeção, a ferramenta Mockup DUE abstrai o processo de mapeamento dos mockups para que o inspetor possa se concentrar exclusivamente na identificação de problemas. A ferramenta também fornece outras funcionalidades específicas como (Rivero et al., 2013a): apontar defeitos de usabilidade e notas diretamente nos mockups; e gerar automaticamente um relatório contendo os defeitos identificados.

\subsection{O Processo de Aplicação das Tecnologias DUE}

A técnica Web DUE e a ferramenta Mockup DUE são aplicadas em conjunto para executar uma inspeção de usabilidade. O processo de aplicação é composto de duas etapas principais: planejamento da inspeção e a detecção de problemas de usabilidade (Rivero et al., 2013a). Durante a etapa de planejamento, os moderadores responsáveis por preparar os mockups devem carregá-los e conectá-los através de links. Um link na ferramenta Mockup DUE serve para ligar um mockup ao outro, e assim simular a interação no sistema. A Figura 1 mostra um exemplo do mapeamento de dois mockups do Sistema de Indicadores ONline $-\mathrm{SION}^{1}$ do estado do Amazonas, que tem por

\footnotetext{
http://sion.secti.am.gov.br/
} 
objetivo apresentar informações sobre indicadores de ciência, tecnologia e inovação à população do estado do Amazonas.

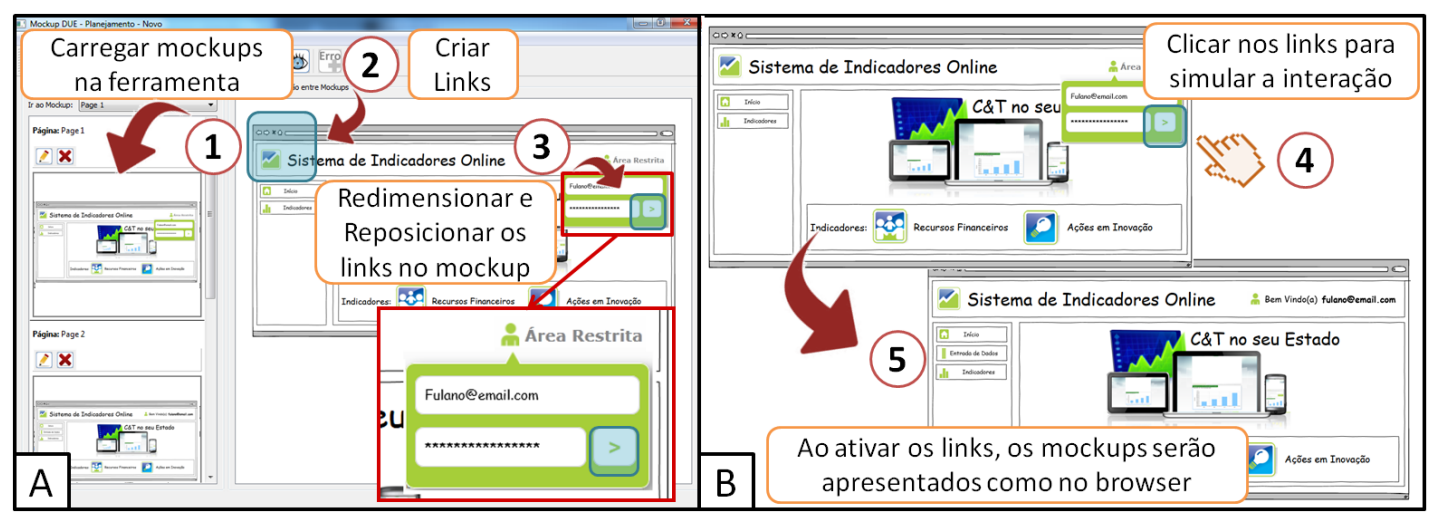

Figura 1. Mapeamento de mockups na ferramenta Mockup DUE.

Durante a etapa de detecção de problemas, os inspetores utilizam os mockups que foram mapeados pelos moderadores e realizam uma inspeção usando a técnica Web DUE. A Parte A da Figura 2 apresenta como a ferramenta Mockup DUE serve de apoio ao processo de identificação de problemas. Desta forma, no Elemento 1 da Figura 2 é mostrada a barra de ferramentas com as funcionalidades disponíveis: (a) cadastrar defeitos e notas, e (b) gerar relatórios de inspeção. Já no Elemento 2 da Figura 2 é mostrada a parte da ferramenta onde são disponibilizados os itens de verificação da técnica Web DUE, que são agrupados segundo as zonas de páginas Web. Finalmente, o Elemento 3 da Figura 2 apresenta a área de interação do sistema avaliado, onde o inspetor terá acesso aos mockups que foram previamente mapeados durante a etapa de planejamento da inspeção. O inspetor pode navegar pelos mockups utilizando os links previamente cadastrados e focar na identificação de defeitos de usabilidade.

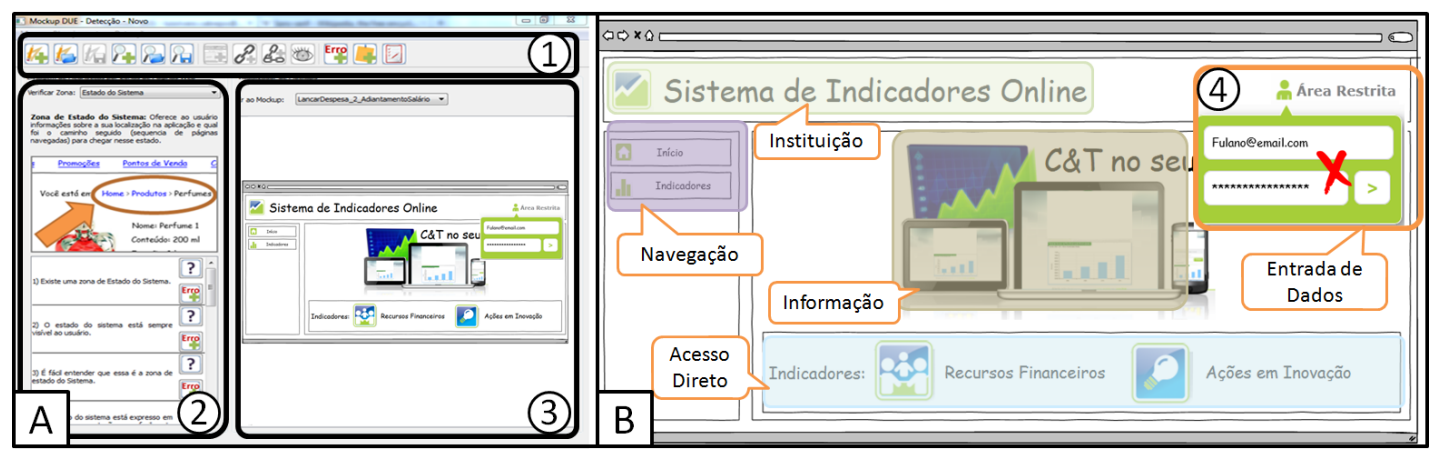

Figura 2. Identificação de defeitos nos mockups usando a técnica Web DUE.

A Figura 2 Parte B exemplifica o processo de identificação de problemas de usabilidade ao aplicar a técnica Web DUE. Inicialmente, o inspetor deve identificar quais são as zonas presentes no mockup. Logo em seguida, para cada zona identificada, o inspetor deverá checar se os itens de verificação da técnica Web DUE estão sendo respeitados pelo mockup avaliado. Por exemplo, na zona de Entrada de Dados, o mockup da Figura 2 Parte B não respeita os itens de verificação descritos na Tabela 1 (ver Subseção 3.1.). Apesar de indicar que dados devem ser preenchidos em cada campo, os campos não possuem nome (ver Item I01). Além disso, no mockup não são 
indicados quais são os campos obrigatórios (ver Item I02) e o símbolo do botão "entrar/login" não é intuitivo (ver Item I03). Finalmente, caso o usuário não possua um usuário ou senha cadastrados, o sistema não fornece apoio para o usuário ter acesso a esses dados (ver Item I04). Ao identificar uma não conformidade em uma das zonas do mockup, o inspetor deverá avaliar se está produzirá um problema de usabilidade. Caso seja esse o caso, o inspetor poderá cadastrar o defeito usando a ferramenta Mockup DUE, indicando o item de verificação que foi violado na técnica Web DUE e a localização exata do defeito (ver Figura 2 Elemento 4).

Para executar uma inspeção completa, todos os mockups do sistema Web devem ser avaliados, checando se os itens de verificação das zonas presentes nos mockups estão sendo respeitados. Durante a avaliação, o inspetor poderá navegar pelos mockups, simulando a interação entre o usuário e o sistema. Finalmente, ao concluir a avaliação, o inspetor poderá gerar relatórios contendo informações sobre: (a) o inspetor, (b) o sistema avaliado, e (c) uma listagem dos problemas de usabilidade identificados e sua localização exata nos mockups. Mais exemplos sobre a aplicação das tecnologias DUE podem ser encontrados em trabalhos anteriores (Rivero e Conte, 2012b, 2012c, 2013b).

\section{Avaliação Experimental das Tecnologias DUE e Evolução}

Para verificar a viabilidade e aplicabilidade do uso das tecnologias propostas, foram executados vários estudos experimentais. A seguir serão descritos cada um destes experimentos, enfatizando seus objetivos, resultados e a evolução das tecnologias DUE.

\subsection{Estudo de Viabilidade da Técnica Web DUE}

O primeiro estudo (Rivero e Conte, 2012b) visou responder a seguinte questão de pesquisa: "É o uso da técnica Web DUE viável em termos do número de problemas de usabilidade identificados e é o tempo bem empregado durante sua aplicação?". A Tabela 2 apresenta o objetivo do estudo usando o paradigma Goal Question Metric GQM (Basili e Rombach, 1988).

Tabela 2. Objetivo do $1^{\circ}$ Estudo Experimental no GQM (Basili e Rombach, 1988).

\begin{tabular}{|l|l|}
\hline Analisar & A técnica de inspeção Web Design Usability Evaluation \\
\hline Com o propósito de & Caracterizar \\
\hline Com respeito a & À viabilidade em termos dos indicadores de eficácia e eficiência ao ser comparada com a técnica WDP. \\
\hline $\begin{array}{l}\text { Do ponto de vista } \\
\text { de }\end{array}$ & Pesquisadores em Engenharia de Software \\
\hline No contexto de & Uma avaliação de usabilidade de mockups de uma aplicação Web executada por inspetores de novatos. \\
\hline
\end{tabular}

Para verificar se a técnica Web DUE era viável, decidiu-se comparar seu desempenho com o da técnica WDP (ver Subseção 3.1.), visto que a técnica WDP foi base para sua criação. Nesse contexto, avaliaram-se dois indicadores amplamente adotados para a investigação da produtividade de novas técnicas de inspeção (Conte et al., 2009): (a) eficácia, razão entre o número de problemas detectados pelo inspetor e o número de problemas existentes conhecidos; e (b) eficiência, razão entre o número de problemas detectados pelo tempo gasto para identificá-los.

Para comparar os indicadores de eficácia e eficiência das técnicas Web DUE e WDP, oito alunos dos cursos de graduação e pós-graduação em Ciência da Computação da Universidade Federal do Amazonas foram convocados a participar do estudo, em que realizaram uma inspeção de usabilidade em cima dos mockups de uma aplicação $W e b$ 
real de um site de cupons. Os alunos assinaram um termo de consentimento livre e esclarecido explicando as atividades que seriam realizadas e preencheram um questionário de caracterização, com o intuito de obter dados sobre sua experiência em: usabilidade, inspeções e atividades de análise e design. Os participantes foram classificados segundo suas respostas como: (a) baixo, caso possuíssem apenas experiência em livros e/ou sala de aula; (b) médio, se além de conhecimentos, tivessem participado das atividades na prática; e (c) alto, caso tivessem aplicado seus conhecimentos na indústria. Com base nas informações, os alunos foram alocados de forma balanceada em uma das duas equipes (Web DUE ou WDP) e receberam treinamentos equivalentes (com os mesmos exemplos) sobre usabilidade, inspeções e a técnica que iriam aplicar. Finalmente, os alunos aplicaram a técnica designada e cada um entregou um relatório contendo a descrição dos possíveis problemas de usabilidade identificados.

Os relatórios entregues foram combinados em um único relatório, contendo todos os possíveis problemas de usabilidade identificados. Esta lista de possíveis problemas foi analisada por uma equipe de inspetores de usabilidade com mais de dois anos de experiência prática na área de usabilidade que determinaram quais problemas eram reais e quais eram falsos positivos. Ao todo, foram identificados 79 problemas de usabilidade reais, e este número serviu para calcular o indicador de eficácia dos inspetores e das técnicas. A Tabela 3 mostra os resultados obtidos no primeiro estudo que apresentam indícios de que a técnica Web DUE poderia aumentar o desempenho dos inspetores na avaliação de mockups de aplicações $W e b$, aumentando o número de problemas de usabilidade identificados, e diminuindo o tempo da inspeção.

Tabela 3. Resultados do $1^{\circ}$ Estudo Experimental (Rivero e Conte, 2012b).

\begin{tabular}{|c|c|c|c|c|c|c|c|c|c|}
\hline & \multirow{2}{*}{$\begin{array}{l}\text { Equipe } \\
\text { Participante }\end{array}$} & \multicolumn{4}{|c|}{ Web DUE } & \multicolumn{4}{|c|}{ WDP } \\
\hline & & 1 & 2 & 3 & 4 & 5 & 6 & 7 & 8 \\
\hline \multirow{3}{*}{$\begin{array}{l}\text { Resultados da } \\
\text { Classificação }\end{array}$} & Experiência em Usabilidade & $\mathrm{M}$ & $\mathrm{B}$ & $\mathrm{B}$ & $\mathrm{A}$ & $\mathrm{B}$ & $\mathrm{B}$ & $\mathrm{A}$ & $\mathrm{M}$ \\
\hline & Experiência em Inspeções & $\mathrm{B}$ & $\mathrm{B}$ & $\mathrm{B}$ & $\mathrm{B}$ & $\mathrm{B}$ & $\mathrm{B}$ & $\mathrm{B}$ & $\mathrm{A}$ \\
\hline & Experiência em Análise e Design & $\mathrm{B}$ & $\mathrm{M}$ & $\mathrm{B}$ & A & $\mathrm{B}$ & $\mathrm{M}$ & $\mathrm{M}$ & $\mathrm{B}$ \\
\hline \multirow{4}{*}{$\begin{array}{l}\text { Resultados da } \\
\text { Inspeção }\end{array}$} & Tempo Gasto (min.) & 100 & 67 & 200 & 160 & 147 & 132 & 124 & 279 \\
\hline & Total de Discrepâncias & 38 & 23 & 27 & 22 & 12 & 33 & 9 & 30 \\
\hline & Falsos Positivos & 19 & 2 & 5 & 4 & 1 & 5 & 0 & 8 \\
\hline & Total de Defeitos & 19 & 21 & 22 & 18 & 11 & 28 & 9 & 22 \\
\hline \multirow{2}{*}{$\begin{array}{l}\text { Resultados dos } \\
\text { Indicadores por } \\
\text { Inspetor }\end{array}$} & Eficácia (\%) & 24,05 & 26,58 & 27,85 & 22,78 & 13,92 & 35,44 & 11,39 & 27,85 \\
\hline & Eficiência (Defeitos por Hora) & 11,40 & 18,81 & 6,60 & 6,75 & 4,49 & 12,73 & 4,35 & 4,73 \\
\hline \multirow{2}{*}{$\begin{array}{l}\text { Resultados dos } \\
\text { Indicadores por } \\
\text { Técnica }\end{array}$} & Eficácia Média (\%) & \multicolumn{4}{|c|}{25,32} & \multicolumn{4}{|c|}{22,15} \\
\hline & $\begin{array}{l}\text { Eficiência Média (Defeitos por } \\
\text { Hora) }\end{array}$ & \multicolumn{4}{|c|}{10,89} & \multicolumn{4}{|c|}{6,58} \\
\hline
\end{tabular}

Para melhor entender os resultados obtidos, foi aplicado um questionário pósinspeção solicitando a opinião dos participantes em termos do uso da técnica Web DUE. Todos os participantes que aplicaram a técnica Web DUE responderam questões abertas sobre as vantagens e dificuldades de utilizar a técnica. A seguir, é apresentado um resumo dos resultados: (a) todos os inspetores concordaram que a técnica era fácil de entender e que as zonas de páginas Web facilitavam a identificação de problemas ao guiar o inspetor; (b) todos os inspetores concordaram que a técnica poderia apoiar a identificação de problemas de usabilidade em mockups de aplicações Web; (c) os inspetores concordaram em que os itens de verificação eram úteis quando combinados com as zonas de páginas Web para facilitar a identificação de problemas; (d) alguns 
inspetores indicaram que alguns itens de verificação da técnica eram amplos ou ambíguos, portanto deviam ser modificados para melhorar o desempenho da técnica; e (e) os inspetores apontaram que o processo de inspeção se torna trabalhoso quando o número de mockups é grande, visto que é necessário simular manualmente a interação entre o usuário e o sistema.

\subsection{Estudo de Viabilidade da Ferramenta Mockup DUE}

Um dos principais resultados do estudo de viabilidade da técnica Web DUE foi que era necessário apoiar o processo de inspeção da técnica, visto que simular a interação do sistema se tornava complicado quando o número de mockups da aplicação aumentava. A necessidade de um apoio ferramental também foi observada na extensão do mapeamento sistemático (ver Subseção 2.2.). Consequentemente, a ferramenta Mockup DUE foi desenvolvida após a realização do estudo de viabilidade da técnica Web DUE com o intuito de facilitar a simulação da interação entre os mockups, e facilitar o uso da técnica Web DUE. Para avaliar a viabilidade do uso da ferramenta Mockup DUE, foi realizado um segundo estudo experimental. O estudo visava responder a seguinte questão de pesquisa: "É o uso da ferramenta Mockup DUE viável em termos de satisfação do usuário e facilidade de uso?". Os resultados deste estudo experimental foram publicados em um trabalho anterior (Rivero e Conte, 2013b).

Foi realizada uma avaliação cooperativa para obter dados qualitativos sobre a facilidade de uso e grau de satisfação dos usuários com a ferramenta Mockup DUE. Nesta avaliação, quatro inspetores experientes com mais de dois anos de experiência em avaliações de usabilidade, exploraram as funcionalidades da ferramenta (ver Subseção 3.3.) enquanto um observador apontava oportunidades de melhoria com base nas suas dificuldades. Enquanto os inspetores realizavam as tarefas de planejamento da inspeção e detecção de problemas, eles falavam em voz alta suas opiniões e dificuldades em relação à ferramenta. Finalmente, os inspetores preencheram um questionário onde informavam as vantagens e desvantagens do uso da ferramenta, assim como sua opinião em termos de satisfação. Analisando as observações e as respostas ao questionário, obteve-se os seguintes resultados: (a) todos os inspetores utilizariam a ferramenta para executar uma nova inspeção de usabilidade; (b) alguns inspetores acharam que a ferramenta poderia providencias maiores informações e dicas sobre seu uso, através de instruções; e (c) alguns inspetores acharam que a ferramenta deveria modificar seu layout e a distribuição dos seus componentes para facilitar seu uso.

\subsection{A Evolução das Tecnologias DUE}

Os resultados do estudo de viabilidade da técnica Web DUE na Tabela 3 mostraram que apesar de permitir a identificação de mais problemas de usabilidade mais rápidamente, a técnica aumentava o número de falsos positivos. Isto é, a técnica Web DUE fazia com que os inspetores apontassem problemas que não são defeitos reais de usabilidade. Além disso, os inspetores corroboraram esta afirmativa como mostra a citação abaixo:

“... alguns itens de verificação avaliam propriedades que não sei dizer se estão totalmente ok ou não, então eu apontei todas as não conformidades." - Inspetor 3.

É necessário melhorar a precisão da técnica Web DUE reduzindo o número de falsos positivos. Com isso, espera se aumentar a confiança nos resultados da inspeção de 
usabilidade, permitindo a identificação de problemas que, ao serem corrigidos, melhorem a qualidade das aplicações Web. Nesse contexto, foram analisadas as descrições dos problemas apontados pelos inspetores no estudo de viabilidade da técnica Web DUE. A Tabela 4 mostra exemplos de falsos positivos apontados pelos inspetores que motivaram a realização de mudanças na técnica.

Tabela 4. Itens de verificação que levaram a apontar falsos positivos.

\begin{tabular}{lll}
\hline ID & Itens de Verificação & Descrição do Problema Apontado \\
\hline D01 & Existe uma zona de entrada de dados do sistema. & "Não existe tal zona." \\
D02 & A interface de usuário facilita a diferenciação dos dados diferentes. & "Não existem dados diferentes." \\
D03 & O sistema oferece um menu de navegação. & "Não vejo um menu." \\
\hline
\end{tabular}

A Figura 3 apresenta as alterações feitas na técnica Web DUE com base na análise dos falsos positivos apontados pelos inspetores usando os itens de verificação da Tabela 4. Na Figura 3, o Elemento A mostra a instrução original da técnica Web DUE, que fez com que os inspetores apontassem defeitos não relacionados com o mockup. Por exemplo, o Item D01 da Tabela 4 levou o inspetor a apontar o defeito da falta de entrada de dados, embora fornecer um meio de entrada de dados dependa da funcionalidade da aplicação. Para evitar que os inspetores apontassem defeitos não relacionados com a aplicação, foi modificada a instrução da técnica Web DUE.

\begin{tabular}{|c|c|}
\hline $\begin{array}{l}\text { Técnica Web DUE v1.0 } \\
\text { Se o mockup não se adere ao item de } \\
\text { verificação um novo problema de } \\
\text { usabilidade foi identificado. }\end{array}$ & $\begin{array}{l}\text { Técnica Web DUE v2.0 } \\
\text { Se o mockup não se adere ao item de verificação, avalie se } \\
\text { tal não conformidade pode ocasionar um problema de } \\
\text { usabilidade na aplicação Web. }\end{array}$ \\
\hline $\begin{array}{l}\text { Nova Instrução para a técnica Web DUE } \\
\text { Avalie se o item de verificação de aplic } \\
\text { estão sendo respeitadas pelo mockup. } \\
\text { Técnica Web DUE v1.0 } \\
\text { A interface de usuário facilita a } \\
\text { diferenciação dos dados diferentes. }\end{array}$ & $\begin{array}{l}\text { kup sendo avaliado. Isto é, se as precondições necessárias } \\
\text { Técnica Web DUE v2.0 } \\
\text { Precondição-Se dados distintos estão sendo solicitados: } \\
\text { Avalie se a interface de usuário facilita a diferenciação dos } \\
\text { dados. }\end{array}$ \\
\hline $\begin{array}{l}\text { Técnica Web DUE v1.0 } \\
\text { O sistema oferece um menu de } \\
\text { navegação. }\end{array}$ & $\begin{array}{l}\text { Técnica Web DUE v2.0 } \\
\text { O sistema oferece um conjunto de links e/ou opções de } \\
\text { navegação para se deslocar de uma página para outra. }\end{array}$ \\
\hline
\end{tabular}

Figura 3. Alterações na técnica Web DUE: (a) instruções reformuladas, (b) precondições acrescentadas e (c) descrição detalhada de itens de verificação.

Foi identificado que os inspetores utilizavam os itens de verificação como regras e não como um guia para a inspeção. Por exemplo, o Item D02 da Tabela 4 foi apontado porque o inspetor achava que fornecer dados diferentes era obrigatório no sistema. Este tipo de falso positivo permitiu identificar que certos itens de verificação só devem ser avaliados se uma precondição é satisfeita. Consequentemente, foi criada uma nova instrução para o uso da técnica, e adicionadas precondições quando necessário como mostra o Elemento B da Figura 3. Finalmente, alguns itens de verificação não forneciam informação suficiente para ajudar na identificação de problemas de usabilidade. Por exemplo, o Item D03 da Tabela 4 foi apontado como um problema, pois não estava suficientemente claro. Apesar da aplicação avaliada fornecer meios de navegação, o inspetor achava que como não eram menus, havia um problema de usabilidade. Consequentemente, para evitar o apontamento deste tipo de falsos positivos, alguns itens de verificação foram mais detalhados como mostra o Elemento C da Figura 3. 
Com relação à nova versão da ferramenta Mockup DUE, foram analisados os dados qualitativos do segundo estudo de viabilidade, incorporando as seguintes melhorias:

Uso de Banco de Dados: Inicialmente a ferramenta usava arquivos XML para armazenar os resultados da inspeção. No entanto, como as imagens dos mockups não ficavam armazenadas em arquivo, isto fazia com que houvesse um risco de perda de informações. Logo, foi integrado SQL na ferramenta, permitindo o armazenamento dos dados (mockups, links, notas e defeitos) em um único arquivo, permitindo salvar e carregar os arquivos de planejamento e detecção de defeitos a qualquer momento.

Estado do Sistema: Como sugerido pelos participantes, foram adicionadas mensagens e caixas de dialogo para informar os usuários das ações da ferramenta.

Elementos de Interface Renomeados: $\mathrm{Na}$ versão inicial da ferramenta, os nomes de alguns elementos de interface confundiam os inspetores. Por exemplo, o botão "Editar" em um mockup sugeria que o inspetor iria editá-lo, porém o que iria editar era a interação entre os mockups. Nesse sentido, foram renomeados elementos de interface que ocasionavam confusão entre os inspetores. Por exemplo, o botão "Editar" mencionado acima foi renomeado para "Editar Mapeamento do Mockup".

Confirmação: Em certas ocasiões a ferramenta executava ações definitivas das quais o usuário poderia se arrepender (por exemplo: apagar um defeito). Na sua nova versão, a ferramenta solicita a confirmação de ações que não podem ser desfeitas.

Relatório de Inspeção: A versão inicial da ferramenta Mockup DUE exportava os dados da inspeção no formato texto. Nesta nova versão, a ferramenta permite criar relatórios completos contendo as seguintes informações: (a) informações sobre o inspetor; (b) informações sobre a aplicação avaliada; (c) imagens dos mockups; e (d) a listagem completa dos defeitos e notas apontados, que aparecem diretamente no mockup avaliado e segundo a localização indicada pelo inspetor.

Suporte a outras técnicas: Visto que a combinação de diferentes técnicas de inspeção pode aumentar o número de problemas de usabilidade identificados (Fernandez et al., 2011), a nova versão da ferramenta Mockup DUE permite utilizar também a técnica Avaliação Heurística (ver Subseção 2.1.). Assim, inspetores experientes nesta técnica podem aplicá-la em mockups Web ou em outro tipo de aplicação, para identificar problemas de usabilidade no início do desenvolvimento.

\subsection{Estudos Experimentais na Indústria e no Contexto Web Móvel}

Com o intuito de avaliar se a técnica Web DUE poderia ser aplicada por engenheiros de software, foi executado um novo estudo experimental. Nesse contexto, a técnica Web DUE foi aplicada por profissionais da indústria com mais de dois anos de experiência no desenvolvimento de aplicações $W e b$. Os profissionais foram divididos em dois grupos e cada um aplicou a técnica Web DUE em conjunto com a ferramenta Mockup DUE. Nesta reaplicação, uma das equipes não recebeu treinamento no uso das tecnologias DUE para verificar se a documentação sobre as tecnologias era suficiente para entender como aplicá-las. Os resultados deste experimento indicaram que as tecnologias DUE poderiam ser aplicadas em conjunto e sem treinamento, sem afetar os resultados da inspeção em termos dos indicadores de eficácia e eficiência. No entanto, 
para facilitar sua utilização, algumas modificações seriam necessárias na documentação: (a) acrescentar informações sobre motivação para o uso das tecnologias; e (b) incluir informações sobre inspeções de usabilidade de um modo geral para contextualizar o uso das tecnologias. Os resultados deste experimento foram publicados em um trabalho anterior (Rivero et al., 2014a).

Para identificar oportunidades de melhoria das tecnologias DUE para o contexto Web móvel, foi executado outro estudo experimental avaliando o seu desempenho e coletando dados sobre os fatores que dificultam a sua utilização. Neste estudo, comparam-se os indicadores de eficácia e eficiência da técnica Web DUE com os da técnica Avaliação Heurística. Os resultados deste estudo indicaram que, visto a técnica Web DUE possuir mais itens de verificação, ela permite identificar mais problemas de usabilidade, porém aumentando também o tempo de inspeção. Dentro das oportunidades de melhoria das tecnologias DUE para o contexto Web móvel, identificou-se a necessidade de criar itens de verificação específicos para avaliar se os mockups da aplicação Web móvel são usáveis em termos do tamanho do dispositivo e o tempo de processamento. Os resultados deste experimento também foram publicados em outro trabalho anteriormente publicado (Kawakami et al., 2014).

\subsection{Avaliando a Melhoria da Qualidade de Aplicações Web Móveis}

Com o intuito de avaliar o efeito da correção dos problemas de usabilidade identificados através do uso das tecnologias DUE, foi realizado um novo estudo experimental. Nesse estudo, uma aplicação Web móvel real (People Bus), nas suas fases iniciais de desenvolvimento, foi inspecionada para identificar problemas de usabilidade que dificultassem sua utilização. A equipe de desenvolvimento do aplicativo utilizou como base os relatórios que um conjunto de inspetores com baixa experiência (alunos do curso de Ciência da Computação nos últimos períodos da faculdade) gerou utilizando a ferramenta Mockup DUE ao aplicar a técnica Web DUE. Desta forma, analisando os problemas de usabilidade identificados e os itens de verificação que foram violados pela aplicação avaliada, a equipe de desenvolvimento do aplicativo sugeriu mudanças de design na interface.

A Tabela 5 descreve alguns dos problemas identificados na aplicação People Bus. Esta aplicação tem por objetivo fornecer informações sobre os ônibus e seu trajeto para os cidadãos da cidade de Manaus (AM). A Figura 4 apresenta parte de uma das telas do aplicativo, antes e depois do reprojeto da interface. Para entender melhor os problemas corrigidos, serão relacionados os elementos de interface destacados na Figura 4 com os itens da Tabela 5. O Elemento A da Figura 4 mostra como foram corrigidos os problemas D01 e D02 da Tabela 5. Foi acrescentado um título para a tela e uma instrução básica do que pode ser feito nela para orientar melhor os usuários. Além disso, as opções de navegação na versão original do aplicativo não estavam organizadas, o que dificultava a navegação. Na nova versão do aplicativo, os elementos aparecem em ordem alfabética e os usuários também possuem uma opção de busca. Por sua vez, o Elemento B da Figura 4 visa demonstrar como o problema D03 na Tabela 5 foi corrigido. Apesar de o usuário cadastrar as paradas de ônibus no aplicativo, o mesmo não podia editá-las e/ou apagá-las se necessário. Para fornecer estas opções ao usuário, foram adicionados botões no aplicativo como mostra o Elemento B da Figura 4. Finalmente, com o intuito de aumentar a utilidade do aplicativo, o defeito D04 foi 
corrigido, modificando o dado a ser cadastrado. Na versão anterior do aplicativo, o usuário não tinha informações sobre a exata localização da parada como mostra o Elemento $\mathrm{C}$ da Figura 4. Na nova versão, o usuário tem o exato endereço do local, corrigindo o defeito apontado pelos inspetores. Para corrigir os problemas apontados ao utilizar as tecnologias DUE, a equipe de desenvolvimento deve fazer sugestões de melhoria que respeitem os itens de verificação da técnica Web DUE, além disso, a equipe de desenvolvimento pode se basear nos exemplos/esclarecimentos (ver Tabela 1) que descrevem propriedades de aplicações usáveis. Finalmente, deve-se verificar que as sugestões de melhoria propostas não entrem em conflito com os outros itens.

Tabela 5. Problemas de usabilidade identificados e itens de verificação.

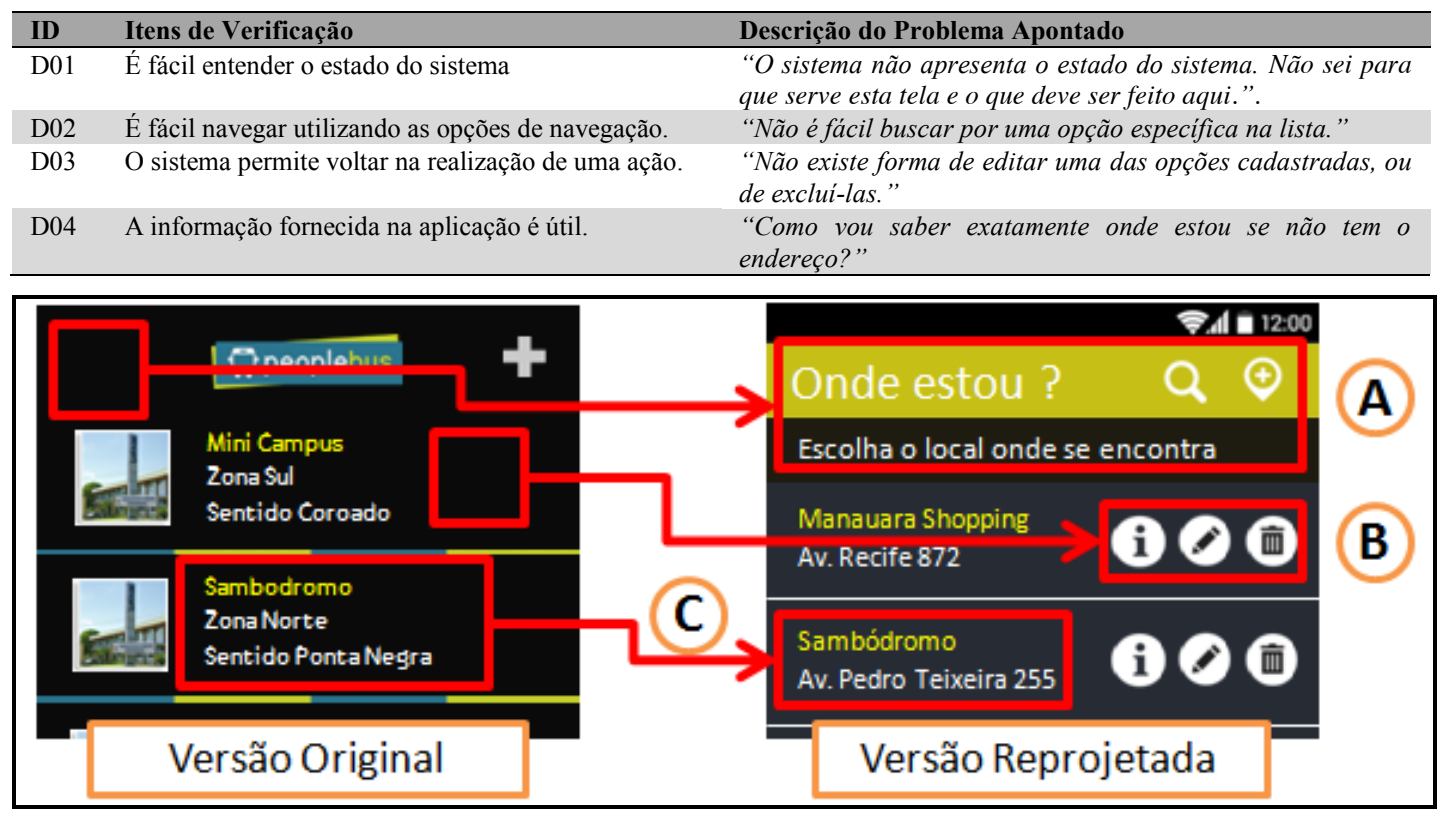

Figura 4. Reprojeto de Interface Corrigindo Problemas de Usabilidade.

Para verificar se do ponto de vista dos usuários, a correção dos problemas apontados ao aplicar as tecnologias DUE aumentava a qualidade da aplicação avaliada, durante a execução do estudo experimental, usuários de diferentes perfis utilizaram ambas as versões da aplicação (original e reprojetada). Durante uma seção de teste, os usuários realizavam as mesmas tarefas nas duas versões e escolhiam a versão que na sua opinião era mais fácil de usar e útil. Os resultados deste estudo indicaram que os usuários conseguiram realizar mais tarefas corretamente na versão reprojetada. Além disso, se os usuários pudessem escolher, baixariam apenas a versão reprojeta. Os resultados deste experimento apontam para o aumento da qualidade e maiores detalhes podem ser obtidos em um trabalho anteriormente publicado (Rivero et al., 2014b).

\section{Conclusões e Trabalhos Futuros}

Este artigo apresentou a motivação para a criação das Tecnologias DUE, e os principais resultados e benefícios desta pesquisa de mestrado. Como mencionado anteriormente, foram propostas novas tecnologias (uma técnica e uma ferramenta), que foram avaliadas experimentalmente e aplicadas em casos reais de desenvolvimento. Além disso, ao publicar os resultados desta pesquisa, foi possível disseminar para a academia e a 
indústria, como aplicar as tecnologias propostas e indicar oportunidades de pesquisa na área de melhoria da qualidade de software através de inspeções de usabilidade. Adicionalmente, os resultados desta pesquisa serviram como referência para a escrita do projeto "Melhorando a Qualidade de Software no Início do Processo de Desenvolvimento de Software" que foi aprovado para financiamento pela Fundação de Amparo à Pesquisa do Estado do Amazonas (FAPEAM), de forma a continuar a aprimorando as tecnologias propostas para a melhoria da qualidade de software.

Os resultados dos estudos experimentais apresentaram indícios da viabilidade do uso das tecnologias DUE para o aumento dos indicadores de eficácia e eficiência de inspeções de usabilidade de aplicações Web. Além disso, ao analisar os dados qualitativos obtidos dos estudos experimentais, foi possível incorporar melhorias nas tecnologias, aplicando-as novamente na indústria e no caso específico de aplicações Web móveis, obtendo resultados satisfatórios. Como trabalho futuro, pretende-se incorporar maiores funcionalidades à ferramenta Mockup DUE e fazer alterações na documentação da técnica Web DUE e seus itens de verificação para que esta possa ser aplicada mais facilmente por engenheiros de software com pouco conhecimento em usabilidade, e sem a necessidade de treinamento. Espera-se com estas medidas, continuar apoiando o desenvolvimento de software de qualidade nas primeiras etapas do processo de desenvolvimento de aplicações $W e b$ a um baixo custo.

\section{Referências}

Basili, V., Rombach, H.: The tame project: towards improvement-oriented software environments. IEEE Transactions on Software Engineering, 14(6), 1988.

Conte, T., Massollar, J., Mendes, E., Travassos, G.: Web usability inspection technique based on design perspectives. IET Software, 3(2), 2009.

de la Vara, J., Wnuk, K., Berntsson-Svensson, R., Sánchez, J., Regnell, B. An Empirical Study on the Importance of Quality Requirements in Industry. In: 23rd International Conference on Software Engineering and Knowledge Engineering - SEKE, 2011.

Fernandez, A., Insfran, E., Abrahao, S.: Usability evaluation methods for the Web: A systematic mapping study. Information and Software Technology, 53(8), 2011.

Fons, J., Pelechano, V., Pastor, O., Valderas, P., Torres, V.: Applying the OOWS model-driven approach for developing Web applications: The internet movie database case study. In: Rossi, G., Schwabe, D., Olsina, L., Pastor, O.: Web Engineering: Modeling and Implementing Web Applications, Springer, 2008.

International Organization for Standardization, ISO/IEC 25010, Systems and software engineering -- SQuaRE - Software product Quality Requirements and Evaluation -System and software quality models, 2011.

Kawakami, G., Rivero, L., Conte, T.: Identifying Improvement Opportunities in Usability Inspection Technologies for the Mobile Web Context though a Controlled Experiment. Proc. XI Experimental Software Engineering Latin Workshop, Chile, 2014. (In Portuguese)

Matera, M., Rizzo, F., Carughi, G.: Web Usability: Principles and Evaluation Methods, In: E. Mendes and N. Mosley, N, Web Engineering, Springer, 2006. 
Nielsen J.: Finding usability problems through heuristic evaluation. Proc. CHI'92, UK, 1992, pp. 373-380.

Polson, P., Lewis, C., Rieman, J., Wharton, C.: Cognitive walkthroughs: a method for theory-based evaluation of user interfaces. International Journal of Man-Machine Studies, 1992.

Rivero, L., Conte, T.: Using an Empirical Study to Evaluate the Feasibility of a New Usability Inspection Technique for Paper Based Prototypes of Web Applications. Journal of Software Engineering Research and Development, 2013b.

Rivero, L., Viana, D., Conte, T.: Mockup DUE: A Tool Support for the Usability Inspection Process of Web Application Mockups. IX Workshop Anual do MPS WAMPS, 2013b. (In Portuguese)

Rivero, L., Barreto, R., Conte, T.: Characterizing Usability Inspection Methods through the Analysis of a Systematic Mapping Study Extension. Latin-american Center for Informatics Studies Electronic Journal, Volume 16, Issue 1, $2013 \mathrm{a}$.

Rivero, L., Conte, T.: Characterizing Usability Inspection Methods through the Analysis of a Systematic Mapping Study Extension. Proc. IX Experimental Software Engineering Latin Workshop, Argentina, 2012a.

Rivero, L., Conte, T.: Improving Usability Inspection Technologies for Web Mockups through Empirical Studies. 25th International Conference on Software Engineering and Knowledge Engineering, 2013a.

Rivero, L., Conte, T.: Usability Inspection for Web Applications Guided by Functionalities. 10th Brazilian Symposium on Human Factors in Computer Systems, Brazil, 2011, pp. 4-5. (In portuguese)

Rivero, L., Conte, T.: Using an Empirical Study to Evaluate the Feasibility of a new Usability Inspection Technique for Paper Based Prototypes of Web Applications. Proc. 26th Brazilian Symposium on Software Engineering, Brazil, 2012b, pp. 81-90.

Rivero, L., Conte, T.: Using the Results from a Systematic Mapping Extension to Define a Usability Inspection Method for Web Applications. Proc. 24th International Conference on Software Engineering and Knowledge Engineering, USA, 2012c.

Rivero, L., Kalinowski, M., Conte, T.: Practical Findings from Applying Innovative Design Usability Evaluation Technologies for Mockups of Web Applications. 47th Hawaiian International Conference on System Sciences, USA, pp. 3054-3063, 2014a.

Rivero, L., Kawakami, G., Conte, T.: Using a Controlled Experiment to Evaluate Usability Inspection Technologies for Improving the Quality of Mobile Web Applications Earlier in their Design. Proc. 28th Brazilian Symposium on Software Engineering, Brazil, 2014b. (Accepted for Publication)

Travassos, G., Shull, F., Fredericks, M., Basili, V.: Detecting defects in object-oriented designs: using reading techniques to increase software quality. Proc. 14th ACM SIGPLAN conference on Object-oriented programming, systems, languages, and applications, USA, pp. 47-56, 1999. 